\title{
Structure Analysis of 16S rDNA Sequences from Strains of Acidithiobacillus ferrooxidans
}

\author{
Hong Peng, Yu Yang, Xuan Li, Guanzhou Qiu, Xueduan Liu, Jufang Huang and Yuehua Hu* \\ School of Minerals Processing and Bioengineering, Central South University, Changsha, 410083, Hunan, China
}

Received 3 October 2005, Accepted 20 December 2005

Four strains of Acidithiobacillus ferrooxidans with different iron oxidation capacity were isolated from different mine drainage stations. The $16 \mathrm{~S}$ rRNA gene of these strains were cloned and sequenced. Based on our sequences analysis on the four strain and the data on the other strains deposited in Genbank, all $\boldsymbol{A}$. ferrooxidans may be classified into three phylogenetic groups. The analysis data showed that nucleotide variables (signature sites) were detected in 21 positions, and most of them were found in the first 800bp from 5, terminal except position 970 and 1375. Interestingly, the first 13 signature sites were located in two main regions:the first region (position 175-234) located in V2 while the second region (position 390-439) were detected in constant region between V2 and V3. Furthermore, the secondary structure and minimal free energy were determined in two regions among strains of three groups. These results may be useful in characterizing the microevolutionary mechanisms of species formation and monitoring in biohydrometallurgical application.

Keywords: Acidithiobacillus ferrooxidans, 16S rDNA sequence, Secondary structure, Signature site

\section{Introduction}

Bacterial leaching of metal sulfides has rapidly increased over the course of the last decade. The process of metal sulfide leaching is commonly referred to as biohydrometallurgy (Rawlings, 2002; Rohwerder et al., 2003) and is now an established biological technique. Nowadays, biohydrometallurgy is employed commercially to recovery low grade copper ores and refractory gold products in China (Yang Songrong et al., 2002) Generally, the predominant microorganisms found in

\footnotetext{
* To whom correspondence should be addressed.

Tel: 86-731-8879815; Fax: 86-731-8879815

E-mail: penghongcsu@yahoo.com.cn
}

these metal leaching systems are acidophilic bacteria that tolerate extremely acidic environments $(\mathrm{pH}<3.0)$. These acidophiles mostly belong to the genus Acidithiobacillus (Kelly and Wood, 2000) and include the species Acidithiobacillus ferrooxidans (A. ferrooxidans), which was the first sulfur or iron oxidizing bacteria to be isolated (Temple and Colmer, 1951;Kelly and Wood, 2000)."

As the first bacterium capable of oxidizing minerals, the genome of $A$. ferrooxidans ATCC23270 was sequenced (http:/ /www.tigr.org) and the molecular genetics and phenotypic aspects of $A$. ferrooxidans were mostly investigated a (Rawlings, 2001; Paulino et al., 2001). Most reports showed that different $A$. ferrooxidans strains were geographically extremely diverse and vary in their physico-chemical conditions in terms of both their genotypic and phenotypic characters (Johnson et al., 2001; Ageeva et al., 2001; Grigorii et al., 2003). The study of the genotypic and phenotypic diversity of strains from different stations played an important role in isolation and identification of species with high bioleaching performance in biohydrometallurgical application.

In this study, the $16 \mathrm{~S}$ rDNA sequences of four wild type $A$. ferrooxidans strains from different environmental stations in China were cloned and sequenced. Furthermore, We analyzed different variable sites (signature sites) of sequences utilizing the complete alignment of four sequences with other sequences in Genbank.

\section{Materials and Methods}

Strains and growth. Four strains of A ferrooxidans (TK, BY, YTW, CMS) were isolated and purified from different sulfide mines drainage. Strains were cultured in $250 \mathrm{ml}$ Erlenmeyer flasks containing $100 \mathrm{ml}$ of Silverman and Lundgren medium 9K (Silverman and Lundgren, 1959) on a shaker at $160 \mathrm{rpm}$ at a temperature of $30^{\circ} \mathrm{C}$. Exponential cultures of $A$. ferrooxidans were used as inoculums (1\%).

Growth rate and ferrous iron oxidation. Enumeration of $A$. ferrooxidans cells were made by direct counting in a Galen III 
microscope. Oxidation of ferrous sulfate by Thiobacillus ferrooxidans was monitored by determining the residual ferrous iron concentration at various intervals. The method of potassium bichromate titration was used. $2 \mathrm{~mL}$ sample was placed in a $50 \mathrm{~mL}$ beaker. Then $10 \mathrm{~mL}$ sulfuric acid and phosphoric acid $(1.5 \mathrm{~mL}$ phosphoric acid + $1.5 \mathrm{~mL}$ sulfuric acid $+7 \mathrm{~mL} \mathrm{H}_{2} \mathrm{O}$ ) was added in beaker, and $0.4 \mathrm{ml}$ diphenysulforic sodium as indicator was added. This sample was titrated with potassium bichromate until the solution changed in color from achromaticity to purple (repeated over three times). A calibration curve of known $\mathrm{Fe}$ (II) concentration was used to calculate the iron concentrations. The concentration of Fe(III) can be calculated by subtracting the $\mathrm{Fe}(\mathrm{II})$ concentration measured at each point from the total iron concentration.

Reaction: $6 \mathrm{Fe}^{2+}+\mathrm{Cr}_{2} \mathrm{O}_{7}^{2-}+14 \mathrm{H}^{+}=6 \mathrm{Fe}^{3+}+2 \mathrm{Cr}^{3+}+7 \mathrm{H}_{2} \mathrm{O}$

DNA extraction and PCR. The pellet was prepared from $150 \mathrm{ml}$ iron medium culture, harvested and washed several times with $\mathrm{H}_{2} \mathrm{SO}_{4}(\mathrm{pH}=2.0)$ to remove precipitation, then was used to maintain the chromosomal DNA by use of the Genomic DNA isolating Kit (SK1201) of Sangon company (Shangha, China) as recommended by the manufacture.The 16S rRNA genes were amplified from all the genomic DNA using universal bacterial primers F27 (5'AGAGTTTGATCMTGGCTCAG3') and R1492 (5'TACGGYTACCTTGTTACGACTT3') (Edwards et al., 1989). The conditions used for PCR were: $10 \times \mathrm{dNTPs}(2 \mathrm{mmol} / \mathrm{l}$ each $) 5$ $\mu \mathrm{l}, 10 \times$ PCR buffer $5 \mu \mathrm{l}$, target DNA $(0.1 \mu \mathrm{g} / \mu \mathrm{l}) 2 \mu \mathrm{l}$, enzyme mixture $($ Taq DNA polymerase : Pfu DNA polymerase $=1: 1) 1 \mu \mathrm{l}$, primers $10 \mathrm{pmol}$ and the reaction mixture made to a final volume $50 \mu \mathrm{l}$ with deionised water. Thermal cycling was as follows: predenaturation at $94^{\circ} \mathrm{C}$ for $5 \mathrm{~min}$, followed by denaturation at $94^{\circ} \mathrm{C}$ for $1 \mathrm{~min}$, annealing at $55^{\circ} \mathrm{C}$ for $1 \mathrm{~min}$ and extension at $72^{\circ} \mathrm{C}$ for 3 min of 30 cycles.

Cloning and sequencing of PCR products. The PCR products were purified by E.Z.N.A ${ }^{\mathrm{TM}}$ Gel extraction Kit (OMEGA), followed as the instructions of the manufacture. The purified products were cloned into PCR2.1 vector using TA cloning kit (Invitrogen).Clones were sequenced by sunbiotech company, which M13 forward and reverse primers were used in sequencing reactions. Sequences have been deposited in GenBank, the accession numbers: TK (DQO0621140, BY (DQO062117), YTW (DQO062116), CMS (DQO062118).

Sequence analysis. Sequences were analyzed and aligned with other sequences deposited in Genbank by ClustalX (Thompson et al., 1997). The secondary structure and minimal free energy were calculated by RNAstructure 4.2 (Mathews et al., 1999). The parameters in study were used in default.

\section{Results}

Isolation and characterization of strains. The four strains were from different mine drainage stations and their econiches varied: TK was from TongKeng zinc mine ores with $\mathrm{pH}$ value 6.60; BY from in BaiYin copper mine drainage with $\mathrm{pH}$ value 3.81; CMS and YTW from low-grade copper mine
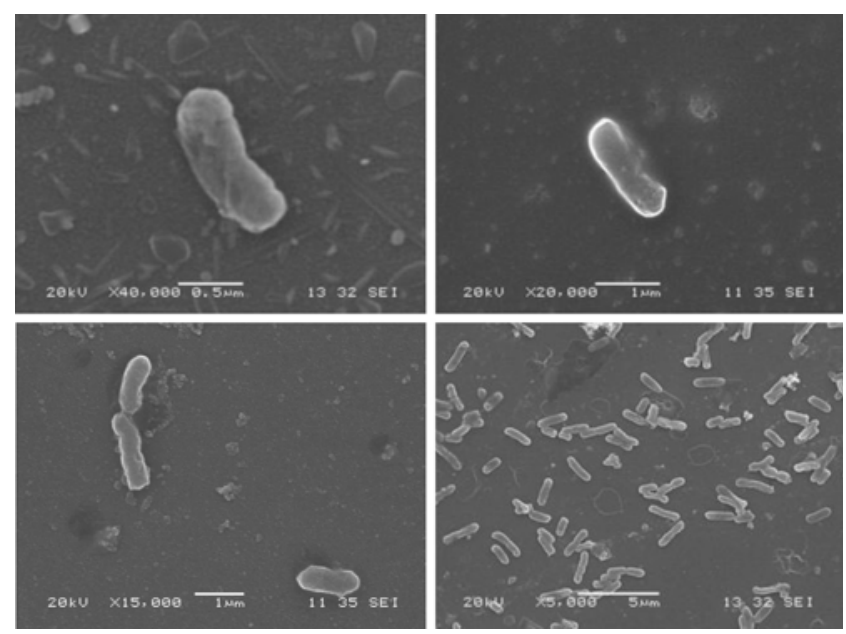

Fig. 1. SEM of strains. Upper left: BY; Upper right: CMS; Down left: TK; Down right: YTW.

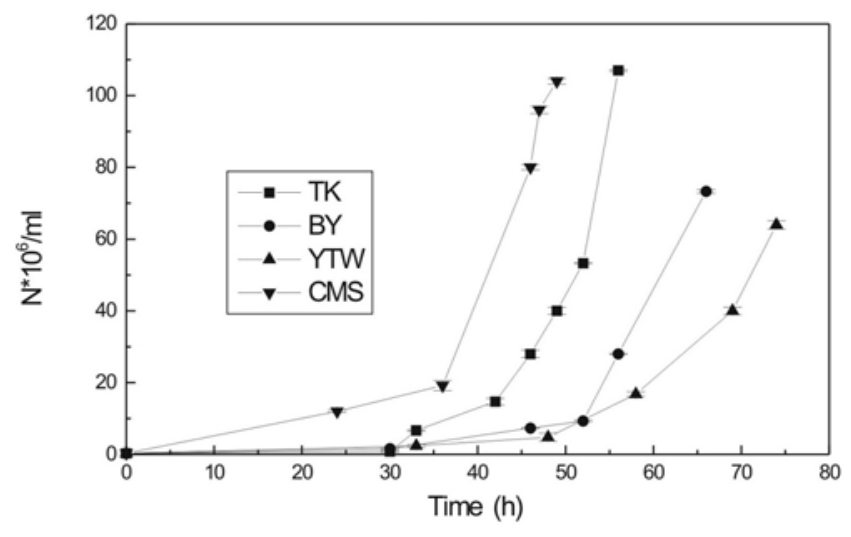

Fig. 2. Growth curves of four strains.

respectively, with $\mathrm{pH}$ value 2.1 and 2.6. Electron micrographs of four strains was shown in Fig. 1. It can be seen that strains were all strait rods. The strains ranged from 1.0 to $2.0 \mu \mathrm{m}$ in length and from 0.4 to $0.6 \mu \mathrm{m}$ in width. Four strains have been identified as $A$. ferrooxidans species according to the 16S rDNA sequences and other phenotypic and physiological data. The 16S rDNA sequences of four strains have been deposited in GenBank (accession numbers: DQO062114, DQO062116 DQO062118). The growth curves of all strains in liquid $9 \mathrm{~K}$ medium were measured and given in Fig. 2. It has been revealed that the growth curves of four strains are different and can be divided into following three items: most actively growing, CMS; the strain TK, medium grown capacity, and those with the lowest growth rate BY and YTW. The ferrous-iron oxidation activity of four strains was shown in Fig. 3. It followed the same order as the growth rate curves. These data reflected the individual phenotypic strain characters that obviously arised from different niches for strains.

Phylogenetic tree of $\boldsymbol{A}$. ferrooxidans strains. Nucleotide sequences $(>1100 \mathrm{nt})$ of the $16 \mathrm{~S}$ rDNA sequences of four $A$. 


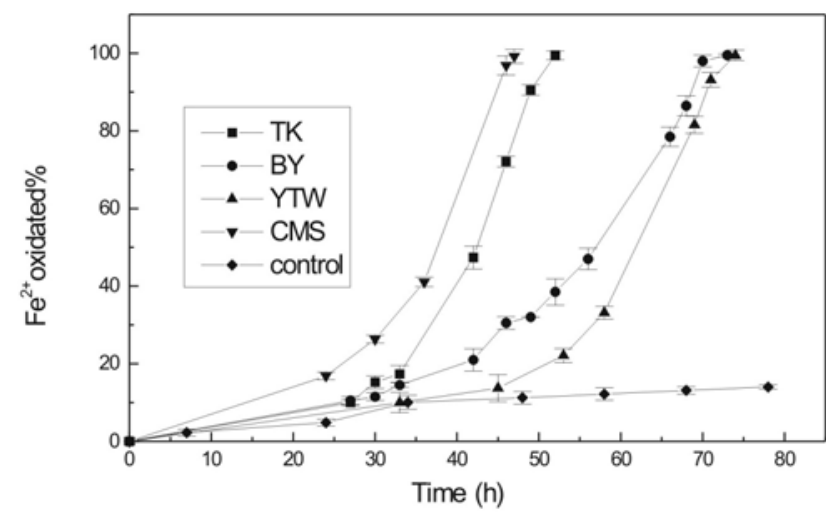

Fig. 3. Oxidation of Ferrous iron of strains (Control: without cells).

Table 1. Strains whose $16 \mathrm{~S}$ rDNA sequences were compared in this study and the accession numbers

\begin{tabular}{cc}
\hline Strain & Accession nunmber. \\
\hline TFI & AF465606 \\
TFD & AF465607 \\
YTW & DQO062116\% \\
TFY & AF465608 \\
TK & DQO062114\% \\
CMS & DQO062118\% \\
DSM9465 & Y11595 \\
NASF-1 & AB039820 \\
WJ13 & AY495953 \\
TF-49 & AJ621559 \\
ATCC23270 & AF465604 \\
BY & DQO062117\% \\
WJ67 & AY495958 \\
SS6 & AJ278721 \\
BRGM1 & AFE457806 \\
CC1 & AFE457804 \\
ATCC19859 & AFE457808 \\
ATCC33020 & AJ278719 \\
DSM 14366 & AJ459804
\end{tabular}

*Total sequences determined in this study; 1 : Partial sequences

ferrooxidans strains were determined. A comparative phylogenetic analysis of the $16 \mathrm{~S}$ rDNA sequences was performed with respect to a large group of $A$. ferrooxidans strains whose 16S rDNA nucleotide sequences are available from the GenBank database (Table 1), while strain DSM 14366 (Acidithobacillus albertensis, the same genus) being as outgroup. From the phylogenetic tree (Fig. 4), all $A$. ferrooxidans strains, including type strain, ATCC23270, may be fall into three phylogenetic groups. The type strain with strain BY and other four strains fell within group I; strain ATCC3302, ATCC19859 and other three strains fell within group II, and strains TFI, TFD, TFY and three wild type strains in this study fell within phylogenetic group III. On the
Table 2. Signature Sites for three groups

\begin{tabular}{cccc}
\hline Position $^{1}$ & GroupI & GroupII & GroupIII \\
\hline 175 & A & G & G \\
178 & G & G & A \\
190 & C & T & T \\
205 & C & T & T \\
206 & G & A & A \\
218 & A & T & T \\
234 & C & A & A \\
394 & A & A & G \\
406 & T & C & C \\
411 & T & T & C \\
419 & A & A & G \\
427 & A & G & G \\
438 & T & T & C \\
587 & G & G & A \\
596 & T & C & C \\
598 & T & G & C \\
787 & G & G & A \\
790 & T & A & T \\
791 & A & G & A \\
970 & A & G & G \\
1375 & G & T & T \\
\hline
\end{tabular}

1: using the type strain ATCC23270 16S rDNA sequences numbering convention

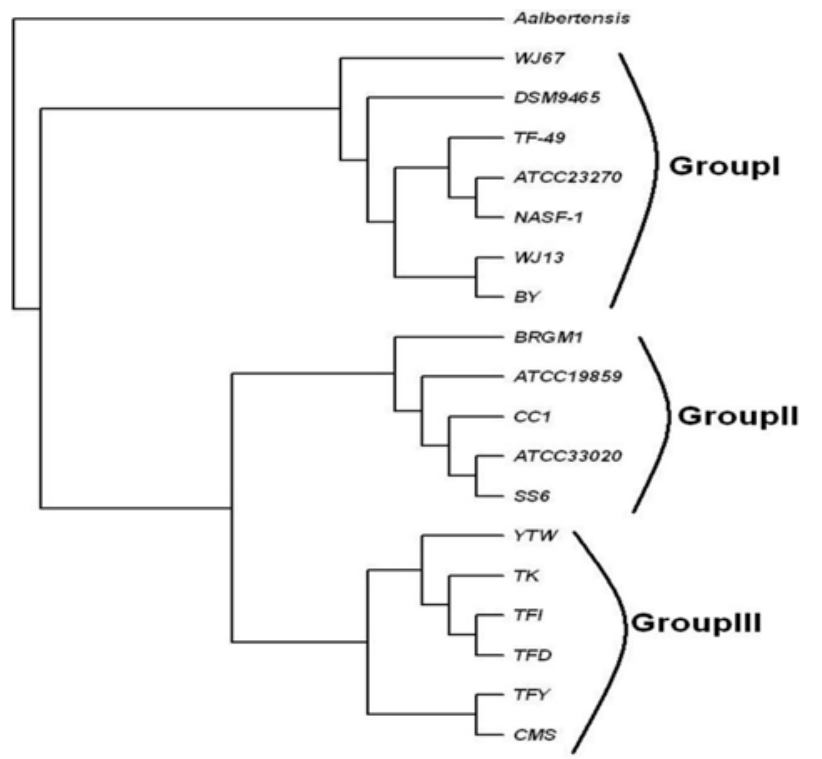

Fig. 4. Phylogenetic tree of various A. ferrooxidans strains based on 16S rDNA sequences. The rectangular cladogram showed the results from ClustalX, with the BOOTSTRAP values from 1000 replicates. A. albertensis DSM 14366 (AJ459804) was used as outgroup.

basis of 16S rDNA nucleotide sequences similarity, strain BY showed sequence similarity of $99.0 \%$ to ATCC23270. Strain 


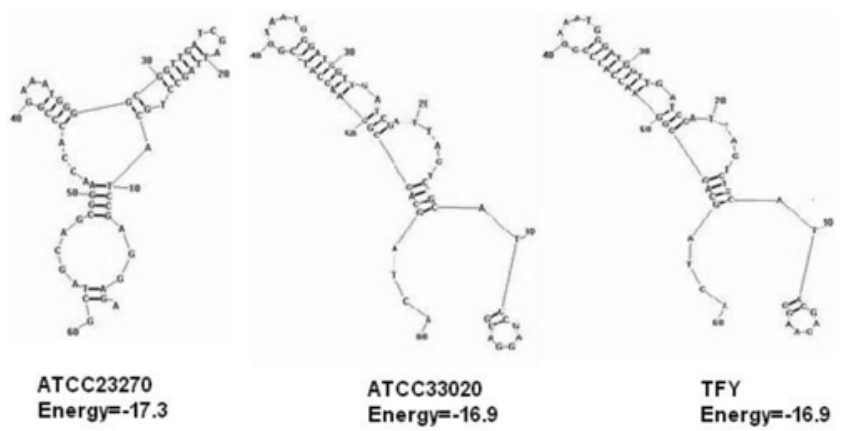

Fig. 5. Secondary structure of the 175-234 region of $16 \mathrm{~S}$ rDNA for three group.
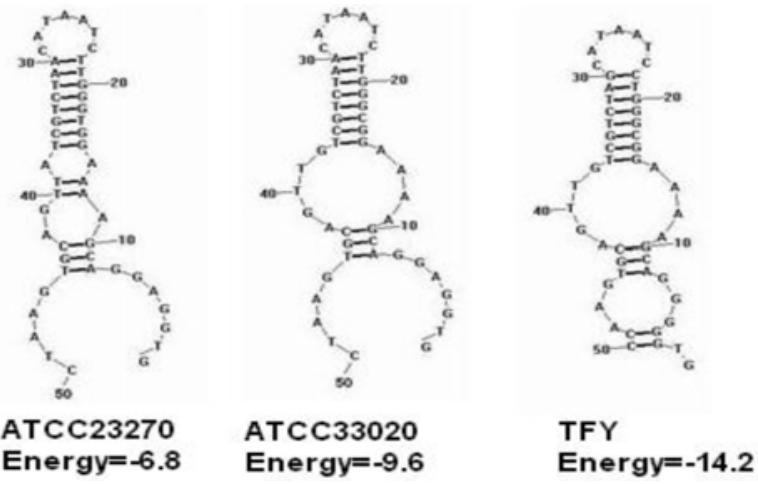

Fig. 6. Secondary structure of the $390-439$ region of $16 \mathrm{~S}$ rDNA for three group.

YTW and CMS is similar with TFD, 99.6\% and 99.1\%, respectively. Strain TK (partial sequence, $1199 \mathrm{bp}$ ) is most similar with TFY with $98.5 \%$ similarity.

Structure analysis of $16 \mathrm{~S}$ rDNA sequences. The complete alignment of nineteen sequences with outgroup showed that the intraspecific variations (signature sites) were not randomly distributed in the $16 \mathrm{~S}$ rDNA sequences of $A$. ferrooxidans (Table 2 \&supporting material). Nucleotide variables were detected in 21 positions and most of them were found in the first 800 bp from 5' terminal except position $970 \& 1375$. Interestingly, the first 13 signature sites were located in two main regions. The first region (position 175-234) located in V2 (variable region) and the second region (position 390-439) were detected in constant region between V2 and V3 (Gray et al., 1984). The secondary structure and minimal free energy of two main regions were calculated by RNAstructure 4.2 (Mathews et al., 1999). To simple the process, the type strain ATCC23270 was used as the representation of GroupI, while ATCC33020 and TFY were designed as GroupII, GroupIII, respectively. The results were demonstrated in Fig. 5 \& Fig. 6. For region 175-234, the structure of ATCC33020 and TFY is similar with same minimal free energy while ATCC23270 has different structure and lower minimal free energy. For region 390-to-439 (Fig. 6), The structure and minimal free energy were different from three groups.

\section{Discussion}

Although all A.ferrooxidans have shared the more-or-less uniform phenotypic being acidophilic, obligately chemolithoautotrophic, Gram-negative rods that use the oxidation of ferrous iron and sulfur for energy generation, they do exhibit considerable genetic variation (Kelly and Wood, 2000). In the past two decades, some work were done to investigate these genotypic variation (Harrison, 1982; Kamimura et al., 2001; Grigorii et al., 2003; HE ZhiGuo et al., 2004). Recently, using RAPD techniques to assess intrespecific variability, similarity coefficients between various isolates which were obtained from different mine ores in China ranged from $44 \%$ to $83 \%$ (He at al., 2004). Grigorii et al. (Grigoriili et al., 2003) reported that A.ferrooxidans strains mainly fell into three phylogentic groups by comparison of nearly complete nucleotide sequences of the 16S rDNA (Grigoriili et al., 2003). Our results showed the similar three phylogentic groups by use of more sequences deposited in Genbank with some minor exceptions. For strain ATCC19859, it is located in GroupII with similar with ATCC33020 but in GroupI with similar with ATCC23270 in their study (Grigoriili et al., 2003). This may be the different outgroup and 16S rDNA sequences in both studies. We only compare different A.ferrooxidans strains without other strains in genus Acidithobacillus. We also compared the environmental conditions of strains to reveal whether the phylogentic groups were correlated with their original geographical sites. The data suggested some certain links between phylogenic similarity and the mineralogical characteristics. For GroupIII, most strains were isolated from copper-zinc or copper ores (TFY, TFI, YTW, CMS, TK) except strain TFD which was isolated from gold-arsenic ores (Grigoriili et al., 2003; Yang et al., 2005). To identify this correlation, it is necessary that largescale investigations of the genotypic features of strains from different environmental sites in relation to the geochemistry of the habitats were performed.

In general, the signature sites in $16 \mathrm{~S}$ rDNA sequences can be used as a molecular marker to distinguish the subspecies (Bertil et al., 1998). In this study, we identified 21 signature sites of 16S rDNA sequences of A.ferrooxidans. Most of variations were detected in the first $800 \mathrm{bp}$ fragments from 5'terminal. Interestingly, for region 175-234, the structure of GroupII was similar with GroupIII which is consistent with the result of phylogentic tree that both Group have high similarity in contrast with GroupI. For region 390-439, the structure of GroupIII has more $\mathrm{G}$ or $\mathrm{C}$ variations than other two groups which was the reason for lower minimal free energy. These signature sites could be used as molecular marker to discern the sub-species of $A$. ferrooxidans for biohydrometallurgical application. The $700 \mathrm{bp}$ fragments from 3'-terminal were very conserved, which may be used to identify A.ferrooxidans species from other species in genus Acidithobacillus. 
Acknowledgment This work was supported by Chinese Science Foundation for Distinguished Group (No. 50321402), National Basic Research Program (973 Program) of P. R. China (No. 2004CB619201).

\section{References}

Ageeva, S. N., Kondrat'eva, T. F. and Karavaiko, G. I. (2001) Phenotypic characteristics of Thiobacillus ferrooxidans strains. Microbiology 70, 186-194.

Bertil, P., Goran, B., Francois, T., Mathias, U. and Karlerik, J. (1998) Molecular evolution of mycoplasma capricolum subsp. capripneumoniae strains, based on polymorphisms in the $16 \mathrm{~S}$ rRNA genes. J. Bateriol. 180, 2350-2358.

Edwards, U., Rogall, T., Blocker, H., Emde, M. and Bottger, E. C. (1989) Isolation and direct complete nucleotide determination of entire genes. Nucleic Acids Res. 17, 7843-7853.

Grigorii, I., Karavaiko, T. P., Turova, T. F., Kondrateva, A. M., Lysenko, T. V., Kolganova, S. N., Ageeva, L. N., Muntyan, T. and Pivovarova, A. (2003) Hylogenetic heterogeneity of the species Acidithiobacillus ferrooxidans. J. Syst. Evol. Microbiol. 53, 113-119.

Gray, M. W., Sankoff, D. and Cedergren, R. J. (1984) On the evolutionary descent of organisms and organelles: a global phylogeny based on a highly conserved structutral core in small subunit ribosomal RNA. Nucleic Acids Res. 12, 58375852 .

http://www.tigr.org

Harrison, A. P. (1982) Genomic and physiological diversity amongst strains of Thiobacillus ferrooxidans and genomic comparison with Thiobacillus thiooxidans. Arch. Microbiol. 131, 68-76.

He, Z. G., Hu, Y. H., Hu, W. X., Zhong, H., Xu, J. and Zhu, M. (2004) Studies of polymorphisms of thiobacillus ferrooxidans using RAPD. Hereditas (Beijing) 26, 69-74.

Johnson, D. B., Rolfe, S., Hallberg, K. B. and Iversen, E. (2001) Isolation and phylogenetic characterization of acidophilic microorganisms indigenous to acidic drainage waters at an abandoned Norwegian copper mine. Environ. Microbiol. 3, 630-637.

Kelly, D. P. and Wood, A. P. (2000) Reclassification of some species of Thiobacillus to the newly designated genera
Acidithiobacillus gen. nov., Halothiobacillus gen. nov. and Thermithiobacillus gen. nov. Int. J. Syst. Evol. Microbiol. 50, 489-500.

Kamimura, K., Wakai, S. and Sugio, T. (2001) Identification of Thiobacillus ferrooxidans strains based on restriction fragment length polymorphism analysis of $16 \mathrm{~S}$ rDNA. Microbioscience 105, 141-152.

Mathews, D. H., Sabina, J., Zuker, M. and Turner, D. H. (1999) Expanded sequence dependence of thermodynamic parameters improves prediction of RNA secondary structure. J. Mol. Biol. 288, 911-940.

Paulino, L. C., Bergamo, R. F., de Mello, M. P., Garcia, O., Manfio, G. P. and Ottoboni, L. M. M. (2001) Molecular characterization of Acidithiobacillus ferrooxidans and A.thiooxidans strains isolated from mine wastes in Brazil. Antonie Van Leeuwenhoek 80, 65-75.

Rohwerder, T., Gehrke, T., Kinzler, K. and Sand, W. (2003) Bioleaching review part A: Progress in bioleaching: fundamentals and mechanisms of bacterial metal sulfide oxidation. Appl. Microbiol. Biotechnol. 63, 239-248.

Rawlings, D. E. (2002) Heavy metal mining using microbes. Anпu. Rev. Microbiol. 56, 65-91.

Yang, S., Xie, J., Qiu, G. and Hu, Y. (2002) Research and application of bioleaching and biooxidation technologies in China. Miner. Engineering 15, 361-363.

Rawlings, D. E. (2001) The molecular genetics of Thiobacillus ferrooxidans and other mesophilic, acidophilic, chemolithotrophic, iron- or sulfur-oxidizing bacteria. Hydrometallurgy 59, 187-201.

Silverman, M. P. and Lundgren, D. C. S. (1959) Study on the chemoautotrophic iron bacterium ferrobacillus ferrooxidans: I. an improved medium and harvesting procedure for securing high cell yield, J. Bacteriol. 77, 642-647.

Temple, K. L. and Colmer, A. R. (1951) The autotrophic oxidation of iron by a new bacterium Thiobacillus ferrooxidans. $J$. Bacteriol. 61, 605-611.

Thompson, J. D., Gibson, T. J., Plewniak, F., Jeanmougin, F. and Higgins, D. G. (1997) The ClustalX windows interface: flexible strategies for multiple sequence alignment aided by quality analysis tools. Nucleic Acids Res. 24, 4876-4882.

Yang, Y., Peng, H., Sun, B., Wang, J.-W. and Hu, Y.-H. (2005) Molecular diversity of the gene encoding Fe(II)-oxidizing enzyme in acidithiobacillus ferrooxidans. Hereditas (Beijing) 27, 787-791. 